\title{
Psychological flexibility and attitudes toward evidence-based interventions by amyotrophic lateral sclerosis patients
}

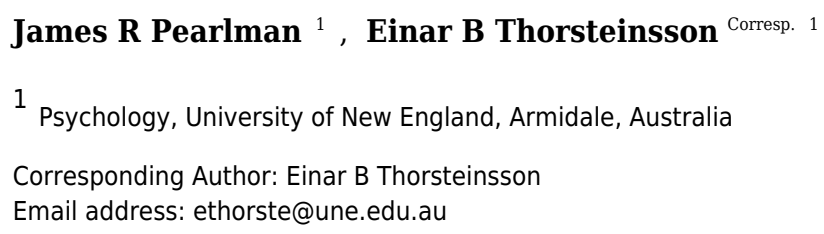

Objective: Declining a percutaneous endoscopic gastrostomy (PEG) or non-invasive ventilation (NIV) by people with Amyotrophic Lateral Sclerosis (ALS) is often contrary to advice provided by health-care-professionals guided by evidence-based principles. This study proposes relational frame theory (RFT) to offer a viable explanation of this phenomenon. Design: Thirty-five people (14 female, 21 male) aged between 34 and 73 years, with ALS, participated in this cross-sectional research. Main Outcome Measures: This research examined the predictive power and interaction effect of psychological flexibility (the fundamental construct of RFT) and psychological well-being on attitudes toward intervention options. Results: Participants with high psychological flexibility reported lower depression, anxiety, and stress, and higher quality of life. In addition, psychological flexibility was predictive of a participant's understanding and acceptance of a PEG as an intervention option. Psychological flexibility was not found to be a significant predictor of understanding and acceptance of NIV. Conclusion: Although the criterion measure had not been piloted or validated outside of the current study and asks about expected rather than actual acceptance, findings suggest that applied RFT may be helpful for clients with ALS. 
9 Authors: James R. Pearlman (ORCID 0000-0002-1571-3797), Einar B. Thorsteinsson (ORCID

10 0000-0003-2065-1989). Affiliation: Psychology, University of New England, Armidale, New

11 South Wales. Australia

12

13

14 Correspondence concerning this article should be addressed to:

15

16 Einar B. Thorsteinsson, $\mathrm{PhD}$

17 Psychology

18 University of New England

19 NSW 2351

20 Australia

21 E-mail: ethorste@une.edu.au 


\section{Abstract}

24 Objective: Declining a percutaneous endoscopic gastrostomy (PEG) or non-invasive ventilation

25 (NIV) by people with Amyotrophic Lateral Sclerosis (ALS) is often contrary to advice provided

26 by health-care-professionals guided by evidence-based principles. This study proposes relational

27 frame theory (RFT) to offer a viable explanation of this phenomenon. Design: Thirty-five people

28 (14 female, 21 male) aged between 34 and 73 years, with ALS, participated in this cross-

29 sectional research. Main Outcome Measures: This research examined the predictive power and

30 interaction effect of psychological flexibility (the fundamental construct of RFT) and

31 psychological well-being on attitudes toward intervention options. Results: Participants with

32 high psychological flexibility reported lower depression, anxiety, and stress, and higher quality

33 of life. In addition, psychological flexibility was predictive of a participant's understanding and

34 acceptance of a PEG as an intervention option. Psychological flexibility was not found to be a

35 significant predictor of understanding and acceptance of NIV. Conclusion: Although the

36 criterion measure had not been piloted or validated outside of the current study and asks about

37 expected rather than actual acceptance, findings suggest that applied RFT may be helpful for 38 clients with ALS. 
40

41

42

43

44

45

46

47

\section{Introduction}

Psychological distress can affect a person's decision-making process, leading to suboptimal outcomes in many instances (Starcke \& Brand 2012). Amyotrophic Lateral Sclerosis (ALS; also referred to as Motor Neurone Disease) is a progressive neurological disease leading to paralysis and ultimately death, most commonly as a result of respiratory failure (Bungener et al. 2005). The psychological impact on the individual can be devastating, with high levels of distress and depression being frequently reported by people with ALS (Pagnini 2013). While there is currently no cure for the neurodegenerative process, there are intervention options available (e.g., percutaneous endoscopic gastrostomy [PEG] or non-invasive ventilation [NIV]) that have been shown to improve the patient's quality of life and prolong the patient's life (Bourke et al. 2006; Kiernan et al. 2011; Leigh et al. 2003). Despite the evidence and bestpractice directives from health authorities, many ALS patients choose not to adopt these intervention options when recommended by health-care professionals (Hogden et al. 2012; Leigh et al. 2003; Orrell 2010).

This decision-making phenomenon gives rise to further questioning regarding possible psychological factors involved. Relational Frame Theory (RFT) is a behaviourist approach in explaining human behaviour as well as internal mental processes (Barnes-Holmes et al. 2004). Research shows support for applied therapies grounded in RFT for the treatment of psychological distress in patients with long-term chronic or terminal illness, such as cancer, multiple sclerosis and HIV (Graham et al. 2016). While there are no published randomised controlled trial results examining applied RFT and people with ALS, this paper proposes that RFT offers a viable account of understanding and acceptance of intervention options in people with ALS. 
64 Amyotrophic Lateral Sclerosis (ALS)

65

66

67

68

69

70

71

72

73

74

75

76

77

The typical ALS patient endures pain and discomfort as a result of severe cramps and twitches; loss of mobility due to weakness and loss of muscle mass; loss of weight and possible malnutrition due to difficulties in getting food to his/her mouth, chewing and swallowing; loss of communication as speech muscles become increasingly affected; and trouble with breathing as diaphragmatic muscles weaken (Leigh et al., 2003). Malnutrition is a critical determinant in the prognosis of an ALS patient and patients who become malnourished increase their risk of death seven-fold (Kiernan et al. 2011; Limousin et al. 2010). Treatment for malnutrition in ALS patients involves the insertion of a small tube through the abdomen wall into the stomach, known as a PEG (Katzberg \& Benatar 2011). This enables caloric intake (liquid feeds and fluids) directly into the stomach, bypassing the mouth and throat (Katzberg \& Benatar 2011).

After a systematic review of non-randomised controlled trials, Orrell (2010) concluded that the use of PEGs in ALS patients has a positive effect on survival. PEGs prevent malnutrition in ALS patients and have been shown to increase mean survival time by 14-months (Limousin et al. 2010). The recommendation from peak health authorities to health-care-professionals caring for ALS patients is to offer a PEG to the patient if rapid weight loss is evident or the patient has difficulties swallowing (Katzberg \& Benatar 2011; Kiernan et al. 2011). It has been estimated that approximately $50 \%$ of patients in the US, and only $23 \%$ of patients in the UK opt for a PEG (Bede et al. 2011). Bede et al. (2011) attributes these differences to variations between health care systems driven by complex local, financial and cultural factors. Berlowitz et al. (2016) reported that $26 \%$ of patients opted for a PEG in their Australian-based study, and interestingly $58 \%$ of patients who used NIV, also opted for a PEG. It is presumed that the influence of health care systems also applies to the Australian data. 

2006). As the ALS patient's respiratory performance declines, the range of associated symptoms decline in mental health (Bourke et al. 2006). NIV offers relief from these symptoms through the use of a small machine, called a Bi-level Positive Airway Pressure (BiPAP), enabling the patient to inhale larger volumes of air and exhale carbon dioxide more effectively (Eng 2006). of use in ALS patients in Australia at 23\%, United Kingdom at 13\%, and United States of America at 20\% (Bede et al. 2011; Berlowitz et al. 2016). Bede et al. (2011) also cites differences between the United States and the United Kingdom to the complex local, financial and cultural drivers of the health care system. As with PEG prevalence rates, it would be presumed that these factors also apply to Australia.

\section{ALS and Psychological Well-being}

Recent research suggests the prevalence of depressive disorders in people with ALS to be approximately 19\%, and, while showing no relationship with functional impairment, depression

has been shown to be strongly correlated with quality of life (Körner et al. 2015; Kurt et al. 2007; McElhiney et al. 2009; Rabkin et al. 2005). well-being and decision-making in people with ALS. Depression has been shown to influence decision-making in patients with chronic illness; depressed patients being three times more likely 
109 to be non-compliant with medical advice, compared to non-depressed patients (Di Matteo et al.

110 2000).

\section{Relational Frame Theory and Understanding and Acceptance of Interventions}

112 Firstly, it should be noted that the terms accept and acceptance used throughout this

113 paper are used in the context of RFT mental processes exclusively and not, for example,

114 confirming the physical adoption of an intervention. A recent study by Greenaway et al. (2015)

115 has elucidated important factors that influence ALS patient's understanding and acceptance of

116 interventions, including: (a) acceptance of the diagnosis and the course of the disease

117 progression, (b) willingness to take action despite having a terminal illness, (c) acceptance of the

118 physical need for an intervention, (d) specific fears relating to the intervention, and (e) timing-

119 factors such as procrastination emanating from a lack of clarity about the likely course of

120 symptoms. The present paper argues that the behaviours observed in the Greenaway et al. (2015)

121 research can be explained by RFT, and that psychological flexibility, as the psychological

122 construct targeted in applied RFT, should be a variable of interest. Furthermore, psychological

123 flexibility could explain a significant portion of the variation between subjects in the

124 understanding and acceptance of intervention options in people with ALS.

125 RFT is grounded in behaviourist principles and aims to move beyond the basic stimulus-

126 response processes of classical and operant conditioning, providing a comprehensive theory of

127 internal processes explaining human cognition, emotion and behaviour as well as offering an

128 explanation for specific psychopathologies (Barnes-Holmes et al. 2004). The processes of RFT

129 are technical in nature and far too extensive for the purpose of explanation in the present paper.

130 Psychological flexibility is the key construct of interest in RFT and is defined by two

131 parts: (a) the processes of experiential avoidance and acceptance, requiring the individual to 
132 make contact with the present moment, and (b) the act of moving through unpleasant

133 psychological reactions toward goals aligned with chosen values (Barnes-Holmes et al. 2004;

134 Fletcher \& Hayes 2005; Hayes 2016; Levin \& Hayes 2009).

135 Greenaway et al. (2015) and Hogden et al. (2012) highlighted acceptance as an

136 influential factor for people with ALS deciding on a PEG or NIV, and the process of acceptance

137 plays an important role in building psychological flexibility (Blackledge \& Barnes-Holmes 2009;

138 Hayes et al. 2006; Levin \& Hayes 2009; Strosahl \& Robinson 2009). Experiential avoidance is

139 said to present in two parts; one, the event-based unpleasant emotion; and two, the action of

140 avoidance (Friman et al. 1998). That is, experiential avoidance manifests in actions taken to avert

141 unpleasant emotions, thoughts, memories or physical sensations (Levin \& Hayes 2009). RFT

142 argues that these unwanted stimuli are a result of overly-dominant comparative frames that is

143 alternative mental representations or frames of reference (Levin \& Hayes 2009). RFT posits that

144 a psychologically flexible individual has the capacity to fully accept internal events, even if

145 unpleasant, and uses strategies (other than avoidance) to move through the discomfort.

146 Greenaway et al. (2015) and Hogden et al. (2012) also explained the importance of

147 perception of choice and control in the context of this decision-making process. RFT claims that

148 behaviour can also be directed by strategic and values-based rules, explained by a frame of

149 coordination between two relational networks (Barnes-Holmes et al. 2004), essentially whereby

150 two or more mental representations are linked or explained to one's self through a self-governed

151 rule or value. Rule-governed behaviour rests on the RFT notion of concept-of-self; requiring the

152 individual not only to behave, but also to use language to assign accurate meaning to one's own

153 behaviour (Barnes-Holmes et al. 2004). According to RFT, sound self-knowledge facilitates

154 effective functioning but omitting the use of language to assign meaning thereby removes the 
155 process of relational framing (Barnes-Holmes et al. 2004). This would be akin to Beck's

156 representation of a maladaptive schema.

157 Psychological flexibility has been shown to have a moderate to strong relationship with

158 general psychological well-being; including quality of life, depression and anxiety (Hayes et al.

159 2006). In addition, longitudinal studies have provided support for the theory that psychological

160 flexibility predicts mental health, and not the reverse (Hayes et al. 2006). In patients with chronic

161 illness, Graham et al. (2016) established that applied RFT is associated with improved outcomes.

162 We are not aware of any published studies examining psychological flexibility in ALS

163 patients. The only published study found of some relevance looked at the longitudinal effect of

164 mindfulness on physical functioning in people with ALS (Pagnini et al. 2015). Pagnini et al.

165 (2015) reported that mindfulness had a positive impact on the patients' functioning, discovering

166 that low-mindfulness patients' physical symptoms declined by $-9.64 \%$ over a four month time

167 period, while high-mindfulness patients' physical symptoms declined by $-2.88 \%$ over the same

168 time. Mindfulness also predicted quality of life, depression, and anxiety. Relationships between

169 quality of life, psychological well-being (depression and anxiety) and physical symptoms were

170 reported to be non-significant.

171 Pagnini et al. (2015) concluded that mindfulness appeared to predict physical symptom

172 progression in ALS patients and proposed that a mind-body interaction is the mechanism

173 responsible. However, given that PEG and NIV interventions were not accounted for in this

174 study, and because these interventions have been shown to influence ALS symptom progression,

175 this proposition should be accepted with caution. 


\section{The Current Study}

177 The aim of the current study was to examine the relationship between psychological

178 flexibility, psychological well-being and understanding and acceptance of interventions in people

179 with ALS, suggesting that applied RFT may have utility with this population. No previous study

180 has investigated these relationships in people with ALS. The present study had two objectives:

181 Firstly, we sought to examine the relationship between psychological flexibility and

182 understanding and acceptance of interventions in people with ALS. Secondly, we set out to

183 examine the role of psychological well-being within the abovementioned relationship, and

184 investigate the possibility that psychological well-being moderates the relationship between

185 psychological flexibility and understanding and acceptance of interventions.

186 H1: Consistent with an RFT account of feelings and behaviour, we expected that high

187 psychological flexibility would be associated with high levels of understanding and acceptance

188 of interventions, for both PEG and NIV. In line with the literature review presented in Hayes et

189 al. (2006), we also expected that high psychological flexibility would also be associated with

190 high psychological well-being (i.e., high quality of life, low depression, low anxiety and low

191 stress).

192 H2: We expected that psychological flexibility and psychological well-being would

193 predict understanding and acceptance of interventions.

194

H3: We expected the relationship between psychological flexibility and understanding

195 and acceptance of interventions to be moderated by psychological well-being. 


\section{Participants}

198 To be eligible for this study, participants had to be aged 18 -years or above and have

199 received a diagnosis of ALS. Verification of diagnosis was not requested of the participant. The

200 final sample consisted of $14(40 \%)$ females and $21(60 \%)$ males $(N=35)$, and participants' age

201 ranged from 34 years to 73 years $(M=55.80, S D=10.48)$. The mean time since first symptoms

202 was 49.14 months $(S D=32.55$ months $)$ and mean time since diagnosis was 26.74 months $(S D=$

20321.66 months). An analysis of country of residence revealed $51.4 \%$ participants lived in

204 Australia, 22.9\% lived in the United Kingdom, 11.4\% lived in the United States of America,

$2055.7 \%$ in Canada, and the rest $8.6 \%$ in other countries. Participation was voluntary and no

206 incentives were offered. All data for this study is available at Figshare.com (Pearlman \&

207 Thorsteinsson 2018).

208 Data Screening

Missing Data. A total of 84 participants were recruited in total; 31 participants withdrew

210 from the study after viewing the information sheet; 15 participants withdrew after only

211 completing the demographic information; 3 participants withdrew after only partially completing

212 the survey (less than $30 \%$ of the survey was completed). The data from these 49 participants was

213 not included in the analysis. Missing data was addressed by calculating a mean score for each

214 subscale (as opposed to total subscale score) and setting the denominator at a minimum of $\mathrm{x}-1$,

215 where $\mathrm{x}$ was the number of items on the subscale.

\section{Materials}

217 Participants were required to complete a demographics sheet include age, gender, country

218 of residence, date of first symptoms and date of diagnosis. Participants were then required to 
219 complete self-report measures designed to assess psychological flexibility, quality of life,

220 depression, anxiety, stress and understanding and acceptance of interventions.

221 Psychological Flexibility. The 16-item version of The Acceptance and Action

222 Questionnaire (AAQ-16) measures psychological flexibility across two factors; (a) willingness;

223 and (b) action (Bond \& Bunce 2003). The first factor, 'willingness', includes seven items

224 designed to measure people's willingness to accept unwanted thoughts and feelings (e.g., "It's ok

225 to feel depressed or anxious.”). The second factor, 'action', includes nine items assessing

226 participants' ability to act in a way that is congruent with their goals and values, even in the

227 presence of unwanted thoughts or feelings (e.g., In order for me to do something important, I

228 have to have all my doubts worked out."). Participants responded to statements by indicating

229 how true they believed the item was for them on a 7-point rating scale ranging from 1 (never

230 true) through to 7 (always true). A mean total score was calculated for each factor. Scores ranged

231 from 1 to 7 where higher scores indicate greater psychological flexibility. Cronbach's alpha

232 reported by Bond \& Bunce (2003) was .87. In the current study, the internal reliability was poor

233 for willingness (Cronbach's alpha $=.47)$ and acceptable for action (Cronbach's alpha $=.69$ ).

234 Quality of Life. The McGill Quality of Life Questionnaire - Revised (MQOL-R) was

235 designed to measure quality of life in people with a terminal illness (Cohen et al. 1995). This

236 instrument includes 14-items to form four subscales: (1) Physical (e.g., "over the past two days,

237 my physical symptoms were:"); (2) Psychological (e.g., "over the past two days, I was nervous

238 or worried:"); (3) Existential (e.g., "over the past two days, I felt good about myself as a

239 person."); and (4) Social (e.g., over the past two days, I felt the relationships with the people I

240 care about were:). Participants were asked to indicate an option that was most true for them on a

241 relevantly labelled 11-point rating scale ranging from 0 (very bad) to 10 (excellent). Sub-scale 
242 scores were calculated by taking the mean score across the items in that sub-scale. A total mean

243 score was calculated by determining the total for each of the 4 sub-scale scores. Total scores

244 range from 0 to 10 where higher scores indicate higher quality of life. Cronbach's alpha is .80

245 (Cohen et al. 1995). Internal reliability in the current study was good (Cronbach's alpha $=.88$ ).

246 Depression, Anxiety and Stress. The 21-item Depression, Anxiety and Stress Scales

247 (DASS-21) was designed to assess features of depression, hyper-arousal and tension-stress in

248 clinical and non-clinical groups (Antony et al. 1998). Participants were presented with statements

249 across these three sub-scales: (1) Depression (e.g., "I couldn't seem to experience any positive

250 feelings at all”); (2) Anxiety (e.g., "I was aware of dryness in my mouth"); and (3) Stress (e.g., "I

251 found it hard to wind down"), and rate how appropriately they believed this item described them

252 over the past week. Responses were captured on a 4-point rating scale ranging from 0 (never) to

2533 (always). Scale scores were generated by calculating the mean total of the respective item

254 scores, where higher scores indicate higher levels of disturbance. Cronbach's alpha for

255 depression, anxiety, and stress was .97, .92 and .95 respectively (Antony et al. 1998). Internal

256 reliability in the current study was good for depression and stress, with Cronbach's alpha at .80

257 and .81 respectively. However, anxiety was only adequate with Cronbach's alpha of .67.

Understanding and Acceptance of Interventions. For the purpose of the present study, a

259 9-item scale measuring understanding and acceptance of PEGs and a 4-item scale measuring

260 understanding and acceptance of NIV were designed. Each item was developed from qualitative

261 findings published in Greenaway et al. (2015). Participants were required to provide self-report

262 responses on a 5-point rating scale in response to questions or statements relating to their current

263 viewpoint or attitude toward the intervention in question (e.g., PEG - "I find the idea of a PEG

264 tube coming from my stomach...” and NIV - "I would use a BIPAP machine if it could prolong 
265 my life.”). Response options were offered on an appropriately labelled 5-point rating scale

266 ranging from 1 (highly unlikely) to 5 (highly likely). Scale scores were generated by calculating

267 the mean total (ranging from 1 to 5), where higher scores indicate higher levels of understanding

268 and acceptance. Item 9 on the PEG-Scale was reverse scored. The internal reliability for both

269 scales was good (PEG-scale Cronbach's alpha $=.80$, NIV-scale Cronbach's alpha $=.82$ ).

270 Procedure

$271 \quad$ Following the submission of a human research ethics application and meeting all

272 conditions in full the University of New England Human Research Ethics Committee approved

273 the project (approval number HE017-069). Advertisements were placed on the MND Association

274 website, MND Association Facebook page, and international ALS online forums.

275 Advertisements included a hyperlink, taking prospective participants directly to the study. Data

276 collection and storage was securely managed through online software (Qualtrics 2017). This

277 online capability enabled participation to people with internet access via a personal computer,

278 tablet or mobile device. The surveys could also be completed using eye-gaze assistive-

279 technology coupled with a tablet. The surveys took approximately 15 minutes to complete.

280 The first page presented to participants was an information sheet detailing participants'

281 rights and addressed ethical considerations. Participation was anonymous and participants were

282 free to withdraw from the study at any time without consequence. Participants were then

283 presented with page titled "Online Implied Consent for Participant", asking the participant to

284 confirm that they had read and understood the information sheet and explaining that clicking on

285 the "PROCEED TO STUDY" button served as implied consent. Withdrawal at any time could

286 be achieved simply by closing down their web browser session. At the completion of the 
287 surveys, participants were thanked for their participation and advised that their responses had 288 been recorded.

\section{Statistical Analyses}

290 Statistical analyses were performed using IBM SPSS Statistical Software version 24. A

291 critical alpha value of .05 was set to test all hypotheses. Bivariate correlational analyses were

292 conducted using Pearson product-moment coefficient calculations. A moderation analysis was

293 performed using Process (Hayes 2018). A power analysis using G*Power 3.1 (Faul et al. 2009)

294 was conducted based on multiple regressions in Pagnini et al. (2015). A sample size of 37

295 participants was recommended based on $R^{2}=.15$, power $=.80, \alpha=.05$, Cohen's $f=0.176$, and

296 six predictors.

297 When examining understanding and acceptance of interventions the main focus was on

298 two factors, PEG and NIV, rather than on individual items. This was consistent with the structure

299 of the hypothesis. However, correlations of individual items from the PEG and NIV factors and

300 other key variables are reported in supplementary material (i.e., tables S1 and S2).

\section{Results}

302 Descriptive Statistics and Correlations

303

To address the possibility of lurking variables, correlations for age, time since first

304 symptoms, and time since diagnosis were run against all variables. Participants' age was not 305 significantly correlated with any variables measured. However, psychological flexibility (action) was associated with time since diagnosis $(r=.45, p<.01$, two-tailed $)$ and time since first 307 symptoms ( $r=.36, p<.05$, two-tailed). That is, as time passes, patients are better able to take 308 action toward goals that are in accord with their chosen values. Descriptive statistics and 309 correlations for all predictor and criterion variables are shown in Table 1. 
311 correlation between psychological flexibility (action) and understanding and acceptance of

312 interventions (PEG). However, no significant relationship between psychological flexibility

313 (action) and understanding and acceptance of interventions (NIV) was found. Similarly, there

314 were no significant relationships between psychological flexibility (willingness) and any other

315 variable.

316 It was also expected that psychological flexibility would be negatively correlated with

317 depression, anxiety and stress, and positively correlated with quality of life. This hypothesis was

318 supported for action, but not for willingness. Thus, these findings suggest that high

319 psychological flexibility, specifically action, is associated with low depression, low anxiety and

320 low stress and high quality of life.

321 Multiple Regression. To test the second hypothesis, a multiple regression analysis was

322 conducted to examine the extent to which psychological flexibility and psychological well-being

323 predicted the understanding and acceptance of interventions in people with ALS, see Table 2.

324 The model overall was a good fit with approximately $38 \%$ of the variance in understanding and

325 acceptance of a PEG being explained by the predictors. Table 2 shows that people with ALS who

326 scored highly on action and quality of life, were expected to have a better understanding and be

327 more accepting of a PEG as an intervention.

328 The second hypothesis suggested that psychological flexibility (willingness and action),

329 depression, anxiety, stress, and quality of life would predict understanding and acceptance of

330 NIV. The fit for the overall model was not significant. Table 3 shows that the relevant

331 regression model was of a moderate strength, with $12 \%$ of the variance in the criterion being 
332 explained by the predictors suggesting that participants who were high in anxiety had higher

333 understanding and acceptance of NIV.

334 Moderation Analysis. A moderation analysis was conducted to test the third hypothesis,

335 that psychological well-being (moderator variable) would moderate the relationship between

336 psychological flexibility (independent variable), and understanding and acceptance of

337 interventions (dependent variable). We therefore expected the interaction effect between the

338 psychological flexibility and the psychological well-being to be significant upon the

339 understanding and acceptance of interventions.

340 Using the consolidated data representing the three variables, the analysis was conducted

341 using Model 1 in Process, using 5000 bootstrapped samples (Hayes 2018). The fit for the model

342 overall was acceptable, $R^{2}=.26, F(3,31)=5.06, p<.01$. However, the interaction effect

343 between psychological flexibility and psychological-well-being on understanding and acceptance

344 of interventions was non-significant $b=-0.01$ [95\% CI $-0.60,0.57]$. Thus, the results did not

345 support the hypothesis.

346

347

348

349

350

351

352

353

354

\section{Discussion}

The findings from the current study may offer some support for the position that an RFT account of behaviour is tenable, when evaluating the attitudes towards a PEG tube in people with ALS. Results supported the hypothesis that psychological flexibility is a significant predictor of understanding and acceptance of a PEG as an intervention for people with ALS. More specifically, the ability for an individual to take action in line with chosen goals and values appears most significant, more so than an individual's willingness to accept unwanted thoughts or feelings. This could be helpful from a clinical perspective with results implying that applied RFT may be helpful for clients with ALS. While building acceptance and lowering experiential 
355 avoidance are generally of prime importance in applied RFT, the current study suggests that the

356 clinician should also be cognisant of developing the client's action skills, enabling the client to

357 make better decisions throughout the course of their illness.

358 The data also suggested that a patient's ability to take action in line with chosen goals is

359 positively related to the time they have had ALS. This relationship may be somewhat intuitive.

360 As time passes, patients may develop a better understanding of the nature of their disease as well

361 as the options available to them that can support their goals. Consequently, they may become

362 better equipped to evaluate options and make decisions, as time progresses. While not included

363 in the scope of this study, the type of ALS (e.g., bulbar-onset, limb-onset) may impact the rate of

364 symptom progression, thereby potentially exerting an influence on the point raised, and as such

365 should be taken into consideration by the reader.

366 The results of the current research also offer some weight to the argument that the

367 conclusions drawn in Pagnini et al. (2015) - that is, that mindfulness predicts ALS symptoms

368 through a mind-body interaction - may be compromised. Instead, these results offer support for

369 the idea that mindfulness, in this case psychological flexibility, may predict a patient's attitudes

370 towards evidence-based treatments that prolong life. Thus, suggesting that the patient's selection

371 of interventions is an extraneous variable in the (Pagnini 2013) research.

372 In line with Hayes et al. (2006), the current study found that high psychological

373 flexibility was shown to be related to lower levels of depression, anxiety and stress as well as

374 higher levels of quality of life. No previous study had examined the possibility that

375 psychological well-being moderated the relationship between psychological flexibility and

376 understanding and acceptance of intervention options. This proposed interaction effect was

377 shown to be non-significant and therefore offers little support for the proposed relationship. 
378 While psychological well-being did not significantly moderate this relationship, it should be

379 noted that quality of life was a significant predictor of understanding and acceptance of a PEG in

380 people with ALS. This relationship should be of interest to clinicians responsible for the delivery

381 of treatment to people with ALS who may be considering intervention options.

382 Our parallel analysis of understanding and acceptance of NIV was less conclusive.

383 Anxiety was the sole significant predictor, however the direction of the relationship suggests

384 somewhat of a paradox from an RFT perspective, with results showing a higher propensity for a

385 participant to be more accepting of NIV when anxiety levels were high. One possible

386 explanation for this relationship may be that the function of NIV (i.e., additional air being

387 supplied to the patient) may be attractive to an anxious patient experiencing shortness-of-breath,

388 hence anxiety may play a causal role. Alternatively, perhaps anxious patients are more likely to 389 readily accept interventions that do not induce further anxiety.

$390 \quad$ Further to the analysis of understanding and acceptance of NIV, if the results observed

391 are a true representation of this population, this would suggest that the nature of NIV decision-

392 making may just different from PEG decision-making. Implementing NIV is: (a) far less invasive

393 than a PEG, (b) easier to administer to the patient than a PEG, (c) a faster process and can be

394 opted for if and when it is needed (unlike a PEG), and (d) easily reversed (unlike a PEG), should

395 the patient wish to opt-out. On the other hand, if the results observed are not a true representation

396 of this population, then factors including measurement error and an insufficient number of

397 participants may also explain the phenomenon observed.

398 Limitations

399 The criterion measure developed for this study had not been piloted or validated outside

400 of the current study. Consequently, the implications that can be drawn from this research may be 
401 somewhat limited. Further to this point, readers should be aware that the generalisations made in

402 the current study were based on the patient's attitudes toward interventions, and in doing so,

403 implying that this would be strongly correlated with the individual's actual behaviour to adopt an

404 intervention. The validity of this assumption should be taken into consideration as a limitation.

405 The predicted effect size of the NIV-based criterion may have been over-estimated, and

406 as such, this segment of the current study had insufficient power. The reason for this smaller

407 effect size may be down to the nature of NIV, as described previously. It should also be noted

408 that the current study fell short of typical recommendations for the minimum number of

409 participants, given six predictor variables, and therefore may have contributed to the study being

410 under-powered.

411 The internal reliability of the measure of psychological flexibility (willingness) was

412 relatively low in the current study. The relative associations may therefore have been

413 underestimated. The literature also refers to similar shortcomings with the AAQ, and cites issues

414 with the complexity of questions and the subtle nature of concepts addressed as potential sources

415 of issue (Bond et al. 2011). Both of these aspects could have contributed to the internal reliability

416 problem in the current study.

417 The current study recruited participants from a number of different countries. Given

418 variation in medical practices (e.g., propensity to perform gastrostomies) and differences in

419 patient's access to resources (e.g., access to BiPAP machines though the public health system)

420 between countries, there may be some sources of variation not accounted for in the current study

421 design. Furthermore, the current study did not allow for any possibility that there might be some

422 cross-cultural influence in the take-up of PEG or NIV in ALS patients. While this is an under- 
423 researched area, there is some evidence to suggest that this might be a factor (Andersen et al.

424 2018).

425 Verification of diagnosis was not sought in the current study, nor did it seek verification

426 for type of ALS. This could potentially impact the data collected (e.g., type of ALS will be

427 coupled with a symptom progression pattern which may, in turn, affect the patient's decisions

428 regarding interventions).

429 The scope of the current study did not include Fronto Temporal Dementia, affecting the 430 executive function, language and personality in $20 \%$ to $50 \%$ of ALS patients (Kiernan et al.

431 2011). Such deficits could have the potential to influence the patient's understanding and

432 acceptance of interventions and possibly the patient's ability to accurately complete the

433 questionnaire items. Finally, the cross-sectional design of this study does not allow us to make

434 causal inferences with regard to our findings.

435

436 Future Research

There are several directions that future research could take that are specific to this research question. The possible causal nature of the relationship between psychological flexibility and understanding and acceptance of interventions should be sought through a longitudinal research design. Alternatively, another research possibility would be to investigate

441 the role of psychological flexibility as a mediator of the relationship between education on PEGs 442 and the understanding and acceptance of PEGs in people with ALS (Hayes 2018; Muller et al.

443 2005). Finally, research to improve the validity of the criterion would be useful.

444 In a broader sense, further research into applied RFT in people with ALS is much needed.

445 The COMMEND Project run by University College London in the United Kingdom is currently 
446 addressing this challenge in their two-phase study, customising an RFT-based therapy for people

447 with ALS and then assessing the treatment's effectiveness within this group.

448

449 Conclusion

450 The present study offers some support for an RFT account of behaviour in explaining

451 understanding and acceptance of intervention options in people with ALS. This research offers a

452 first step toward developing an empirical base for applied RFT to be used with ALS patients, as

453 it is with other chronic and terminally ill populations (Graham et al. 2016). Furthermore, the

454 action factor of psychological flexibility has been highlighted to be of some importance,

455 especially in relation to the decision to opt for a PEG. This study should serve as grounds for

456 further research to elucidate the mechanisms involved, and to remind therapists who choose to

457 use an applied RFT approach, of the importance of action skills as they formulate a strategy to

458 improve psychological well-being and quality of life in people with ALS.

459

460 


\section{Acknowledgements}

462 The author would like to acknowledge the effort and support of the Motor Neurone

463 Disease Association of New South Wales for its assistance in recruiting participants.

464

465 
Andersen PM, Kuzma-Kozakiewicz M, Keller J, Aho-Oezhan HE, Ciecwierska K, Szejko N, Vázquez C, Böhm S, Badura-Lotter G, and Meyer T. 2018. Therapeutic decisions in ALS patients: Cross-cultural differences and clinical implications. Journal of Neurology 265(7):1600-1606. DOI 10.1007/s00415-018-8861-4

469
470

471

472

473

Antony MM, Cox BJ, Enns MW, and Swinson RP. 1998. Psychometric properties of the 42-Item and 21-item versions of the Depression Anxiety Stress Scales in clinical groups and a community sample. Psychological Assessment 10(2):176-181. DOI 10.1037/1040-3590.10.2.176

Barnes-Holmes Y, Barnes-Holmes D, McHugh L, and Hayes SC. 2004. Relational Frame Theory: Some implications for understanding and treating human psychopathology. International Journal of Psychology and Psychological Therapy 4(2):355-375.

Bede P, Oliver D, Stodart J, van den Berg L, Simmons Z, O'Brannagain D, Domenico Borasio G, and Hardiman 0. 2011. Palliative care in amyotrophic lateral sclerosis: A review of current international guidelines and initiatives. Journal of Neurology, Neurosurgery and Psychiatry 82:413-418. DOI 10.1136/jnnp.2010.232637

Berlowitz DJ, Howard ME, Fiore Jr JF, Vander Hoorn S, O'Donoghue FJ, Westlake J, Smith A, Beer F, Mathers S, and Talman P. 2016. Identifying who will benefit from non-invasive ventilation in amyotrophic lateral sclerosis/motor neurone disease in a clinical cohort. Journal of Neurology, Neurosurgery and Psychiatry 87:280-286. DOI 10.1136/jnnp-2014-310055

Blackledge JT, and Barnes-Holmes D. 2009. Core processes in Acceptance and Commitment Therapy. In: Blackledge JT, Ciarrochi, J., \& Deane, F. P., ed. Acceptance and Commitment Therapy: Contemporary theory, research and practice. Brisbane, Australia: Australian Academic Press, 4158.

Bond FW, and Bunce D. 2003. The role of acceptance and job control in mental health, job satisfaction, and work performance. Journal of Applied Psychology 88(6):1057-1067. DOI 10.1037/00219010.88.6.1057

Bond FW, Hayes SC, Baer RA, Carpenter KM, Guenole N, Orcutt HK, Waltz T, and Zettle RD. 2011. Preliminary psychometric properties of the Acceptance and Action Questionnaire-II: A revised measure of psychological inflexibility and experiential avoidance. Behavior Therapy 42(4):676688. DOI 10.1016/j.beth.2011.03.007

Bourke SC, Tomlinson M, Williams TL, Bullock RE, Shaw PJ, and Gibson GJ. 2006. Effects of noninvasive ventilation on survival and quality of life in patients with amyotrophic lateral sclerosis: A randomised controlled trial. The Lancet Neurology 5(2):140-147. DOI 10.1016/S14744422(05)70326-4

Bungener C, Piquard A, Pradat P, Salachas F, Meininger V, and Lacomblex L. 2005. Psychopathology in amyotrophic lateral sclerosis: A preliminary study with 27 ALS patients. Amyotrophic Lateral Sclerosis 6:221-225. DOI 10.1080/14660820510037863

Cohen SR, Mount BM, Strobel MG, and Bui F. 1995. The McGill Quality of Life Questionnaire: a measure of quality of life appropriate for people with advanced disease. A preliminary study of validity and acceptability. Palliative Medicine 9:207-219. DOI 10.1177/026921639500900306

Di Matteo MR, Lepper HS, and Crogan TW. 2000. Depression is a risk factor for noncompliance with medical treatment: Meta-analysis of the effects of anxiety and depression on patient adherence. Archives of Internal Medicine 160:101-107. DOI 10.1001/archinte.160.14.2101

Eng D. 2006. Management guidelines for motor neurone disease patients on non-invasive ventilation at home. Palliative Medicine 20:69-79. DOI 10.1191/0269216306pm1113oa

Faul F, Erdfelder E, Buchner A, and Lang A. 2009. Statistical power analyses using G*Power 3.1: Tests for correlation and regression analyses. Behavior Research Methods 41(1149-1160). DOI 10.3758/brm.41.4.1149

Fletcher L, and Hayes SC. 2005. Relational Frame Theory, Acceptance and Commitment Therapy, and a functional analystic definition of mindfulness. Journal of Rational-Emotive and CognitiveBehavior Therapy 23(4):315-336. DOI 10.1007/s10942-005-0017-7

Friman PC, Hayes SC, and Wilson KG. 1998. Why behavior analysts should study emotion: The example of anxiety. Journal of Applied Behavior Analysis 31, 137-156(1):137-156. DOI 10.1901/jaba.1998.31-137 
520

521

522

523

524

525

526

527

528

529

530

531

532

533

534

535

536

537

538

539

540

541

542

543

544

545

546

547

548

549

550

551

552

553

554

555

556

557

558

559

560

561

562

563

564

565

566

567

568

569

570

571

572

573

574

Graham CD, Gouick J, Krahé C, and Gillanders D. 2016. A systematic review of the use of Acceptance and Commitment Therapy (ACT) in chronic disease and long-termconditions. Clinical Psychology Review 46:46-58. DOI 10.1016/j.cpr.2016.04.009

Greenaway LP, Janssen A, Goldstein LH, Martin NH, Al-Chalabi A, Lawrence V, and Leigh PN. 2015. Accepting or declining non-invasive ventilation or gastrostomy in amyotrophic lateral sclerosis: patients' perspectives. Journal of Neurology 262:1002-1013. DOI 10.1007/s00415-0157665-z

Hayes AF. 2018. Introduction to mediation, moderation and conditional process analysis: A regressionbased approach. New York, NY: Guilford Press.

Hayes SC. 2016. Acceptance and Commitment Therapy, Relational Frame Theory, and the third wave of behavioral and cognitive therapies - Republished article. Behavior Therapy. DOI 10.1016/j.beth.2016.11.006

Hayes SC, Luoma JB, Bond FW, Masuda A, and Lillis J. 2006. Acceptance and Commitment Therapy: Model, processes and outcomes. Behaviour Research and Therapy 44:1-25. DOI 10.1016/j.brat.2005.06.006

Hogden A, Greenfield D, Nugus P, and Kiernan MC. 2012. What influences patient decision-making in amyotrophic lateral sclerosis multidisciplinary care? A study of patient perspectives. Patient preference and adherence 6:829. DOI 10.2147/PPA.S37851

Katzberg HD, and Benatar M. 2011. Enteral tube feeding for amyotrophic lateral sclerosis/motor neuron disease. Cochrane Database of Systematic Review - Intervenion(1). DOI 10.1002/14651858.CD004030.pub3

Kiernan MC, Vucic S, Cheah BC, Turner MR, Eisen A, Hardiman O, Burrell JR, and Zoing MC. 2011. Amyotrophic Lateral Sclerosis. Lancet 377:942-955. DOI 10.1016/S0140-6736(10)61156-7

Körner S, Kollewe K, Abdulla S, Zapf A, Dengler R, and Petri S. 2015. Interaction of physical function, quality of life and depression in Amyotrophic lateral sclerosis: characterization of a large patient cohort. BMC Neurology 84(15):1-8. DOI 10.1186/s12883-015-0340-2

Kurt A, Nijboer F, Matuz T, and Kubler A. 2007. Depression and Anxiety in Individuals with Amyotrophic Lateral Sclerosis; Epidemiology and Management. CNS drugs 21 (4):279-291.

Leigh PN, Abrahams S, Al-Chalabi A, Ampong M, Goldstein LH, Johnson J, Lyall R, Moxham J, Mustfa N, Rio A, C Shaw, and Willey E. 2003. The management of Motor Neurone Disease. The Journal of Neurology, Neurosurgery and Psychiatry 74(4):32-47. DOI 10.1136/jnnp.74.suppl_4.iv32

Levin M, and Hayes SC. 2009. ACT, RFT, and Contextual Behavioral Science. In: Blackledge JT, Ciarrochi, J., \& Deane, F. P., ed. Acceptance and Commitment Therapy: Contemporary theory, research and practice. Brisbane, Australia.: Australian Acedemic Press, 1-40.

Limousin N, Blasco H, Corcia P, Gordon PH, De Toffol B, Andres C, and Praline J. 2010. Malnutrition at the time of diagnosis is associated with a shorter disease duration in ALS. Journal of the Neurological Sciences 297 36-39. DOI 10.1016/j.jns.2010.06.028

McElhiney MC, Rabkin JG, Gordon PH, Goetz R, and Mitsumoto H. 2009. Prevalence of fatigue and depression in ALS patients and change over time. Journal of Neurology Neurosurgery and Psychiatry 80:1146-1149. DOI 10.1136/jnnp.2008.163246

Muller D, Judd CM, and Yzerbyt VY. 2005. When moderation is mediated and mediation is moderated. Journal of Personality and Social Psychology 89(6):852-863.

Orrell RW. 2010. Motor neuron disease: Systematic reviews of treatment for ALS and SMA. British Medical Bulletin 93:145-159. DOI 10.1093/bmb/ldp049

Pagnini F. 2013. Psychological wellbeing and quality of life in amyotrophic lateral sclerosis: A review. INTERNATIONAL JOURNAL OF PSYCHOLOGY 48(3):194-205. DOI 10.1080/00207594.2012.691977

Pagnini F, Phillips D, Bosma CM, Reece A, and Langer E. 2015. Mindfulness, physical impairment and psychological well-being in people with amyotrophic lateral sclerosis. Psychology \& Health 30(5):503-517. DOI 10.1177/1359105314530453

PearIman JR, and Thorsteinsson EB. 2018. Adoption of evidence-based interventions by amyotrophic lateral sclerosis patients and psychological flexibility. DOI 10.6084/m9.figshare.6159182.v1

Qualtrics. 2017. Qualtrics Research Suite [Computer software]. Provo, UT. Retrieved from http://www.qualtrics.com 
575 Rabkin JG, Albert SM, Del Bene ML, O'Sullivan I, Tider T, Rowland LP, and Mitsumoto H. 2005.

576

577

578

579

580

581

582
Prevalence of depressive disorders and change over time in late-stage ALS. Neurology 65(1):6267. DOI 10.1212\%2F01.wnl.0000167187.14501.0c

Starcke K, and Brand M. 2012. Decision making under stress: A selective review. Neuroscience and Biobehavioral Reviews 36:1228-1248. DOI 10.1016/j.neubiorev.2012.02.003

StrosahI KD, and Robinson PJ. 2009. Teaching ACT: To whom, why and how. In: Blackledge JT, Ciarrochi, J., \& Deane, F. P., ed. Acceptance and Commitment Therapy: Contemporary theory, research and practice. Brisbane, Australia: Australian Academic Press, 59-85. 


\section{Table $\mathbf{1}$ (on next page)}

Matrix of Intercorrelations, Means, Standard Deviations and Ranges for Psychological Flexibility, Depression, Anxiety, Stress, Quality of Life, and Understanding and Acceptance of Interventions (Percutaneous Endoscopic Gastrostomy and Non-Invasive Ventila 
1 Table 1

2 Matrix of Intercorrelations, Means, Standard Deviations and Ranges for Psychological Flexibility, Depression, Anxiety, Stress, 3 Quality of Life, and Understanding and Acceptance of Interventions (Percutaneous Endoscopic Gastrostomy and Non-Invasive 4 Ventilation).

\begin{tabular}{|c|c|c|c|c|c|c|c|c|}
\hline Variable & 1 & 2 & 3 & 4 & 5 & 6 & 7 & 8 \\
\hline 1. Willingness & - & .17 & -.27 & -.16 & -.05 & .05 & .10 & .20 \\
\hline 2. Action & & - & $-.49 * *$ & $-.45 * *$ & $-.54 * *$ & $.40^{*}$ & $.59 * *$ & .21 \\
\hline 3. Depression & & & - & $.44 * *$ & $.45^{* *}$ & $-.60 * *$ & -.32 & -.25 \\
\hline 4. Anxiety & & & & - & $.38 *$ & -.33 & -.32 & .19 \\
\hline 5. Stress & & & & & - & $-.69 * *$ & $-.45 * *$ & -.16 \\
\hline 6. Quality of Life & & & & & & - & $.54 * *$ & .27 \\
\hline 8. UAI (NIV) & & & & & & & & - \\
\hline$M$ & 4.05 & 4.75 & 1.90 & 1.73 & 2.01 & 5.99 & 3.58 & 4.21 \\
\hline$S D$ & 0.82 & 0.79 & 0.52 & 0.46 & 0.49 & 1.68 & 0.82 & 0.87 \\
\hline Range Possible & $1-7$ & $1-7$ & $1-4$ & $1-4$ & $1-4$ & $0-10$ & $1-5$ & $1-5$ \\
\hline Range Actual & $2.43-5.86$ & $3.56-5.86$ & $1.00-3.14$ & $1.00-2.86$ & $1.14-3.14$ & $1.17-9.02$ & $1.89-5.00$ & $1.00-5.00$ \\
\hline
\end{tabular}

Note. UAI (PEG) = Understanding and Acceptance of Interventions (Percutaneous Endoscopic Gastrostomy). UAI (NIV) $=$

Understanding and Acceptance of Interventions (Non-Invasive Ventilation). To reduce the family-wise error rate when examining these correlations without a priori hypothesis a criterion of $r>.40$ is suggested.

$* p<.05$, two-tailed. $* * p<.01$, two-tailed. 


\section{Table 2 (on next page)}

Predicting Understanding and Acceptance of Intervention (for Percutaneous Endoscopic Gastrostomy) from Psychological Flexibility and Psychological Well-being. 
1

2 Table 2

3 Predicting Understanding and Acceptance of Intervention (for Percutaneous Endoscopic

4 Gastrostomy) from Psychological Flexibility and Psychological Well-being.

\begin{tabular}{lcccccc} 
& \multicolumn{7}{c}{$\mathrm{CI}_{95 \%}$ for $b$} & & $s r^{2}$ \\
\cline { 3 - 4 } Predictor & $b$ & Lower & Upper & $\beta$ & $r$ & .00 \\
\cline { 1 - 1 } Psychological flexibility & & & & & & \\
$\quad$ Willingness & 0.04 & -0.25 & 0.33 & 0.04 & .10 & .16 \\
$\quad$ Action & 0.54 & 0.17 & 0.91 & 0.52 & .59 & .03 \\
Depression & 0.35 & -0.26 & 0.97 & 0.23 & -.32 & .00 \\
Anxiety & -0.08 & -0.65 & 0.50 & -0.04 & -.32 & .00 \\
Stress & 0.16 & -0.55 & 0.86 & 0.10 & -.45 & .00 \\
Quality of life & 0.25 & 0.04 & 0.46 & 0.51 & .54 & .11 \\
\hline
\end{tabular}

5 Note. Fit for model $R^{2}=.49$, Adjusted $R^{2}=.38, F(6,28)=4.40, p<.01$. The squared semi-

6 partial $\left(s r^{2}\right)$ correlation given is the squared part-correlation given from SPSS. The $r$ given is the 7 zero-order correlation given from SPSS. 


\section{Table 3(on next page)}

Predicting Understanding and Acceptance of Intervention (for Non Invasive Ventilation) - Raw Data Version from Psychological Flexibility and Psychological Well-being. 
2 Table 3

3 Predicting Understanding and Acceptance of Intervention (for Non Invasive Ventilation) - Raw

4 Data Version from Psychological Flexibility and Psychological Well-being.

\begin{tabular}{|c|c|c|c|c|c|c|}
\hline \multirow[b]{2}{*}{ Predictor } & \multirow[b]{2}{*}{$b$} & \multicolumn{2}{|c|}{$\mathrm{CI}_{95 \%}$ for $b$} & \multirow[b]{2}{*}{$\beta$} & \multirow[b]{2}{*}{$r$} & \multirow[b]{2}{*}{$s r^{2}$} \\
\hline & & Lower & Upper & & & \\
\hline \multicolumn{7}{|c|}{ Psychological flexibility } \\
\hline Willingness & 0.19 & -0.18 & 0.56 & 0.18 & .20 & .03 \\
\hline Action & 0.27 & -0.20 & 0.74 & 0.25 & .22 & .04 \\
\hline Depression & -0.26 & -1.04 & 0.52 & -0.16 & -.25 & .01 \\
\hline Anxiety & 0.86 & 0.14 & 1.59 & 0.36 & .19 & .15 \\
\hline Stress & 0.11 & -0.79 & 1.00 & 0.06 & -.16 & .00 \\
\hline Quality of life & 0.14 & -0.13 & 0.40 & 0.26 & .27 & .03 \\
\hline
\end{tabular}

5 Note. Fit for model $R^{2}=.28$, Adjusted $R^{2}=.12, F(6,28)=1.80, p>.05$. The squared semi-

6 partial $\left(s r^{2}\right)$ correlation given is the squared part-correlation given from SPSS. The $r$ given is the

7 zero-order correlation given from SPSS. The transformed data showed the same pattern of

8 findings (beta values) but a higher Adjusted $R^{2}(.19)$, thus the more conservative raw findings are

9 examined due to the small sample size.

10

11 